\title{
Is gabapentin an effective treatment choice for hemicrania continua?
}

\author{
Roderick C. Spears
}

Received: 16 February 2009/ Accepted: 20 April 2009/Published online: 13 May 2009

(C) Springer-Verlag 2009

\begin{abstract}
The objective of this study is to examine the efficacy of gabapentin for the treatment of hemicrania continua (HC) in cases where patients had difficulty tolerating indomethacin due to adverse effects. A retrospective chart review of nine patients with $\mathrm{HC}$ between October 2006 and February 2008. Inclusion criteria included men and women age 18 or above presenting to the headache center with a headache that meets International Headache Society criteria for HC including a response to indomethacin, but were not able to continue on indomethacin secondary to adverse effects. Four patients report being pain free, three patients report a $50-80 \%$ reduction of pain, one patient reports a $10 \%$ reduction of pain, and one patient reports no change in pain. Seven out of nine patients demonstrated at least a $50 \%$ reduction of pain on gabapentin, four patients becoming completely pain free.
\end{abstract}

Keywords Hemicrania continua - Gabapentin . Efficacy · Pain free

\section{Introduction}

Hemicrania continua $(\mathrm{HC})$ is an uncommon primary headache disorder characterized by a continuous, mild to moderate intensity, unilateral headache [1-4]. Most patients will experience superimposed exacerbations of more severe pain, often associated with ipsilateral autonomic symptoms. Diagnostic criteria for $\mathrm{HC}$ are as follows: [5].

Headache for greater than 3 months fulfilling the following criteria:

R. C. Spears $(\bowtie)$

Cleveland Clinic, Cleveland, OH, USA

e-mail: spearsr2@ccf.org
All of the following:

Unilateral pain without side shift, daily and continuous, without pain-free periods, moderate intensity, but with exacerbations of severe pain.

At least one of the following autonomic features occurs during exacerbations and ipsilateral to the side of pain: Conjunctival injection and/or lacrimation, nasal congestion and/or rhinorrhea, ptosis and/or miosis.

A complete response to therapeutic doses of indomethacin.

Finally, not attributed to another disorder.

There is a group of patients that meets the above criteria for $\mathrm{HC}$, but has to discontinue indomethacin secondary to adverse effects, primarily involving the gastrointestinal (GI) tract. The side effects can range from gastritis to severe bleeding, patients may also develop an allergy to indomethacin, see an elevation of blood pressure, or an exacerbation of asthma symptoms.

Gabapentin is often used in an off label fashion for the treatment of neuropathic pain and is well tolerated from a side effect profile. Marinano da Silva, Alcantara, Bordini, and Speciali reported a case of a unilateral headache similar to $\mathrm{HC}$ that was responsive to gabapentin [6].

\section{Methods}

Institutional Review Board approval was obtained for the study. A retrospective chart review of nine patients with HC between October 2006 and February 1, 2008. Inclusion criteria included men and women age 18 or above presenting to the headache center with a headache that meets IHS criteria for $\mathrm{HC}$, including a response to indomethacin, but were unable to continue on indomethacin 
secondary to adverse side effects. Exclusion criteria included anyone under the age of 18 and anyone not meeting IHS criteria for $\mathrm{HC}$ including an adequate trial at therapeutic doses of indomethacin and response to indomethacin. Patients with concomitant disease, specifically involving pain were not excluded, although every effort was made to enroll patients where $\mathrm{HC}$ was their primary complaint. The primary end point was at least a $50 \%$ reduction of pain. This was decided since the purpose of this study was to examine gabapentin as an alternative therapy, indomethacin remains the gold standard. The secondary end point was becoming pain-free. Points of measurement included the duration of disease which is considered to be from onset to completion of the study, temporal profile, duration of therapy, range of dose, and concurrent use of other medications for pain, concomitant disease, especially related to pain, reduction of pain, and the side effects of gabapentin. Onset of relief on gabapentin was not measured.

\section{Results}

This study included eight women and one man (see Table 1). The age ranged from 36 to 74 years. The duration of therapy on gabapentin ranged from 3 months to 13 months with eight out of nine patients remaining on gabapentin at the end of the study. One patient discontinued after reaching 3,600 $\mathrm{mg}$ a day due to lack of efficacy. The other doses ranged from $600 \mathrm{mg}$ to $3,600 \mathrm{mg}$ per day of gabapentin. Two patients reported concurrent use of other medications for pain control. One patient also on fentanyl patch $50 \mathrm{mcg}$ every $72 \mathrm{~h}$ reported an $80 \%$ reduction in pain after reaching 2,400 $\mathrm{mg}$ of gabapentin. The second patient was taking topiramate and became pain free upon reaching $600 \mathrm{mg}$ of gabapentin.

The seven patients that achieved the primary end point and reported a 50-80\% reduction of pain had doses ranging from 900 to $3,600 \mathrm{mg}$ per day. The one man reported a $50 \%$ reduction of pain and reached the maximum dose of $3,600 \mathrm{mg}$ of gabapentin per day.

Four patients achieved the secondary end point and reported being completely pain free on gabapentin. The dose ranges for these patients were, $600 \mathrm{mg}, 900 \mathrm{mg}$, $1,500 \mathrm{mg}$, and $1,800 \mathrm{mg} /$ day respectively. It should be noted that one of the four patients was also taking topiramate, which has been reported to be efficacious for $\mathrm{HC}$ [7, 8].

Two patients failed to achieve either the primary or secondary end points. The patient, who reported no benefit, responded to indomethacin on two separate occasions but developed GI bleeding as a result of indomethacin. The other patient reported a $10 \%$ reduction of pain.

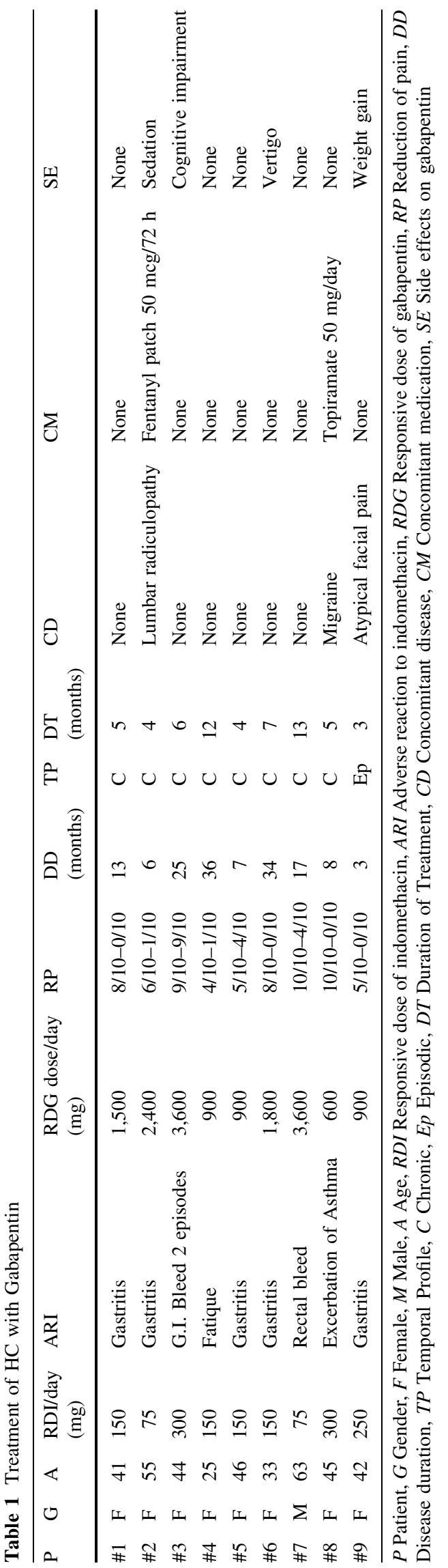


The major side effects reported on gabapentin include sedation, cognitive impairment, depression, and weight gain. None of the patients chose to discontinue gabapentin as a result of the above side effects.

\section{Discussion}

HC was first described in 1981 and officially named by Sjaastad and Spierings in 1984 [9, 10]. There are now more than 100 reports of $\mathrm{HC}$ in the literature. $\mathrm{HC}$ was originally believed to be a rare primary headache disorder but more recent evidence suggests that $\mathrm{HC}$ is not rare, just under recognized [2]. In the clinical setting, $\mathrm{HC}$ is characterized by a unilateral headache that is continuous and of mild to moderate severity. The pain is usually reported as dull, aching, or pressure-like pain without associated symptoms. Exacerbations or more severe pain, lasting anywhere from 20 min to several days, are experienced in the majority of those with $\mathrm{HC}$, and when present are associated with one or more autonomic symptoms on the ipsilateral side. Migrainous features, such as photophobia, phonophobia, nausea, and vomiting commonly occur during exacerbations of pain. Many patients will experience a sensation of a foreign body in their eye similar to sand or an eyelash on the affected side. The pathophysiology of HC is not known, some scientist believe it is a subtype of migraine, while others believe it is more closely related to the trigeminal autonomic cephalalgias (TAC). It was hoped that functional brain imaging would help to clarify our understanding of $\mathrm{HC}$, but it revealed that $\mathrm{HC}$ is a distinct headache syndrome, not belonging to the migraine or TAC group. The scans revealed activation of the contralateral posterior hypothalamus and ipsilateral dorsal rostral pons, as well as activation of the ipsilateral ventrolateral midbrain, extending over the red nucleus and substania nigra and the bilateral pontomedullary junction [11]. These areas have been previously demonstrated to be the sites of activation in the TACs and migraine, ipsilateral activation hypothalamic activation in short-lasting unilateral neuralgiform headache attacks with conjunctival injection and tearing (SUNCT) and cluster; in HC the findings are reversed. HC was not included in the first edition of the ICHD-I [12], but is included within the other primary headaches section of the second edition of the ICHD-II [5].

Hemicrania continua presents as two temporal profiles: an episodic form with distinct headache phases separated by periods of pain-free remissions, and a chronic form in which the headache persists without remission for years [3]. HC is chronic from onset in $53 \%$ of patients; the disorder began in the episodic form and evolved into the chronic form in $35 \%$, and it begins and remains episodic in $12 \%$ of patients [2].
Hemicrania continua may be incorrectly diagnosed as cluster headache. This can occur by focusing exclusively on the ipsilateral autonomic features that accompany the painful exacerbations of HC. Similarly, focusing on the associated photophobia, phonophobia, nausea, and vomiting that may occur during exacerbations of $\mathrm{HC}$ may lead to misdiagnosing $\mathrm{HC}$ as migraine. An absolute response to therapeutic doses of indomethacin helps distinguish $\mathrm{HC}$ from chronic migraine. Autonomic features on the ipsilateral side, which are present during exacerbations of $\mathrm{HC}$, are at times also present during acute attacks of migraine. $\mathrm{HC}$ is also distinguished from cluster headache and migraine by the presence of a continuous baseline headache that is usually unilateral and mild to moderate in severity. The associated features of photophobia, phonophobia, nausea, and vomiting, as well as the ipsilateral autonomic features of cluster are absent with the continuous baseline pain of $\mathrm{HC}$.

Some patients are unable to tolerate indomethacin due to GI distress, renal disease, or concurrent anticoagulation therapy, and alternative types of therapy must be considered. Peres and Silberstein demonstrated in a study using cyclooxygenase-2 inhibitors (COX-2), efficacy of celecoxib and rofecoxib, the latter of which has since been removed from the market. They found $60 \%$ of patients who received celecoxib and $33 \%$ who received rofecoxib experienced a complete response [13]. There is one case in the literature of a unilateral headache similar to $\mathrm{HC}$ that did not respond to indomethacin, and many other standard headache agents, but was responsive to gabapentin at 1,200 mg per day. That patient had complete pain relief after 17 years of continuous headache. [6]. There have also been two separate reports of melatonin being efficacious for $\mathrm{HC}$, one case reported by Spears [14], and three cases by Rozen [15]. There have also been four cases in the literature of patients responding to topiramate, one by Camarda et al. [16], one by Matharu et al. [7], and two cases by Brighina et al. [8]. Rozen also reported one case responsive to verapamil [17].

Gabapentin is an antiepileptic drug that is approved for use in the adjunctive treatment of partial seizures with and without secondary generalization in patients over 12 years of age. Gabapentin is also indicated as adjunctive therapy in the treatment of partial seizures in pediatric patients age 3-12 years of age. Gabapentin is also indicated for the management of post herpetic neuralgia in adults. Benefit has been reported in gabapentin treatment for hypobaric hypoxia-induced headache, high altitude headache, nummular headache, SUNCT syndrome, chronic cluster headache, post-dural puncture headache, and primary thunderclap headache just to mention headaches disorders [18-26]. 
The mechanism by which gabapentin exerts its analgesic action is unknown, but in animal models of analgesia, gabapentin prevents allodynia (pain related behavior in response to a normally innocuous stimulus) and hyperalgesia (exaggerated response to painful stimuli). Gabapentin also decreases pain related responses after peripheral inflammation [27].

The mechanism by which gabapentin exerts its anticonvulsant action is unknown, but in animal test systems designed to detect anticonvulsant activity, gabapentin prevents seizures. Gabapentin is structurally related to the neurotransmitter gamma-aminobutyric acid (GABA) but does not modify GABA a or GABA b radioligand binding, it is not converted metabolically into GABA or a GABA agonist, and it is not an inhibitor of GABA uptake or degradation. Gabapentin was tested in radioligand binding assays and did not exhibit affinity for a number of other common receptor sites including benzodiazepine, glutamate, $N$-methyl-D-aspartate (NMDA), quisqualate, kainate, strychnin-insensitive glycine, alpha 1, alpha 2, or beta adrenergic, adenosine A1 or A2, cholinergic muscarinic or nicotinic, dopamine D1 or D2, histamine H1, serotonin S1 or S2, opiate mu, delta or kappa, cannabinoid 1, voltage sensitive calcium channel sites labeled with nitrendipine or diltiazem, or at voltage sensitive sodium channel sites labeled with batrachotoxinin A 20 alpha benzoate. Gabapentin also did not alter the cellular uptake of dopamine, noradrenaline or serotonin.

One patient reported an $80 \%$ reduction of pain on 2,400 mg of gabapentin but was also on the fentanyl patch. There have been reports in the literature of enhancement of pain control on the combination of opioids and gabapentin [28, 29]. It should be noted that this patient was taking opioids for a concomitant condition. This patient met the primary endpoint, but the concomitant use of an opioid is a confounding factor.

\section{Conclusions}

This study demonstrates that gabapentin is a good choice for alternative therapy in the treatment of HC. Seven out of nine patients demonstrated at least a $50 \%$ reduction of pain on gabapentin, four patients becoming completely pain free. The results of the study are confounded in two patients that were taking concomitant medications specifically fentanyl patch and topiramate. Another potential confounding factor is that a proton pump inhibitor was not initiated when patients had difficulty tolerating indomethacin. Clinically it may be prudent to first try initiating therapy with a proton pump inhibitor while continuing indomethacin prior to changing to gabapentin. $\mathrm{HC}$ is a particularly difficult headache disorder to treat if the patient is not able to tolerate indomethacin, therefore alternative therapies are being sought to provide relief to this patient population. The results of this study should be validated in a double-blinded randomized control trial.

Conflict of interest None.

\section{References}

1. Bordini C, Antonaci F, Stover LJ, Scharader H, Sjaastad O (1991) "Hemicrania continua": a clinical review. Headache 31:20-26. doi:10.1111/j.1526-4610.1991.hed3101020.x

2. Peres MFP, Silberstein SD, Nahmias S, Shechter AL, Rozen TD, Young WB (2001) Hemicrania continua is not that rare. Neurology 57:948-951

3. Newman LC, Lipton RB, Solomon S (1994) Hemicrania continua: ten new cases and a review of the literature. Neurology 44:2111-2114

4. Goadsby PJ, Lipton RB (1977) A review of paroxysmal hemicranias, SUNCT syndrome and other short-lasting headaches with autonomic features, including new cases. Brain 120:193-209. doi:10.1093/brain/120.1.193

5. Headache Classification Committee of the International Headache Society (2004) The international classification of headache disorders, 2nd ed. Cephalalgia 24(Suppl 1):9-160. doi:10.1111/ j.1468-2982.2003.00824.x

6. Mariano da Silva H, Alcantara MC, Bordini CA, Speciali JG (2002) Strictly unilateral headache reminiscent of hemicrania continua resistant to indomethacin but responsive to gabapentin. Cephalalgia 22:409-410. doi:10.1046/j.1468-2982.2002.00361.x

7. Matharu MS, Bradbury P, Swash M (2005) Hemicrania continua: side alternation and response to topiramate. Cephalalgia 26:341344. doi:10.1111/j.1468-2982.2005.01034.X

8. Brighina F, Palermo A, Cosentino G, Fierro B (2007) Prophylaxis of Hemicrania Continua: two new cases effectively treated with topiramate. Headache 47(3):441-443

9. Medina JL, Diamond S (1981) Cluster headache variant: spectrum of a new headache syndrome. Arch Neurol 38:705-709

10. Sjaastad O, Spierings EL (1984) Hemicrania Continua: another headache absolutely responsive to indomethacin. Cephalalagia 4:65-70. doi:10.1046/j.1468-2982.1984.0401065.x

11. Matharu MS, Goadsby PJ (2005) Functional brain imaging in hemicrania continua: implications for nosology and pathophysiology. Current Pain Headache Reports 4:133-140

12. Headache Classification Committee of the International Headache Society (1988) Cephalalgia 8(Suppl 7):1-96. doi:10.1046/ j.1468-2982.1988.0801001.x

13. Peres MF, Silberstein SD (2002) Hemicrania Continua responds to cyclooxygenase-2 inhibitors. Headache 42:530-531. doi: 10.1046/j.1526-4610.2002.02131.x

14. Spears RC (2006) Hemicrania Continua: a case in which a patient experienced complete relief on melatonin. Headache 46(3):524527. doi:10.1111/j.1526-4610.2006.00386_5.x

15. Rozen TD (2006) Melatonin responsive hemicrania continua. Headache 46(7):1203-1204. doi:10.1111/j.1526-4610.2006.00514_1.x

16. Camarda C, Camarda R, Monastero R (2008) Chronic paroxysmal hemicrania and hemicrania continua responding to topiramate: two case reports. Clin Neurol Neurosurg 110(1):88-91. doi: 10.1016/j.clineuro.2007.09.002

17. Rozen TD (2006) Verapamil-responsive hemicrania continua in a patient with episodic cluster headache. Cephalalgia 26(3):351353 
18. Jafarian S, Abolfazli R, Gorouhi F, Rezaie S, Lotfi J (2008) Gabapentin for prevention of hypobaric hypoxia-induced headache: randomized double blind clinical trial. J Neurol Neurosurg Psychiatry 79(3):321-323

19. Jafarian S, Gorouhi F, Salimi S, Lotfi J (2007) Low-dose gabapentin in treatment of high altitude headache. Cephalalgia 27(11):1274-1277. doi:10.1111/j.1468-2982.2007.01387.x

20. Grosberg BM, Solomon S, Lipton RB (2007) Nummular headache. Curr Pain Headache Rep 11(4):310-312. doi:10.1007 /s11916-007-0209-1

21. Rocha Filho PA, Rabello GD, Galvao AC, Fortini I, Calderaro M, Carrocini D (2007) Gabapentin in the treatment of SUNCT syndrome. Arq Neuropsiquiatr 65(2B):503-505. doi:10.1590/ S0004-282X2007000300027

22. Cohen AS (2007) Short-lasting unilateral neuralgiform headache attacks with conjunctival injection and tearing. Cephalalgia 27(7):824-832. doi:10.1111/j.1468-2982.2007.01352.x

23. Schuh-Hofer S, Israel H, Neeb L, Reuter U, Arnold G (2007) The use of gabapentin in chronic cluster headache patients refractory to first-line therapy. Eur J Neurol 14(6):694-696. doi:10.1111/ j.1468-1331.2007.01738.x
24. Trucco M (2007) Nummular headache: another case treated with gabapentin. J Headache Pain 8(2):137-138. doi:10.1007/s10194007-0375-X

25. Lin YT, Sheen MJ, Huang ST, Horng HC, Cherng CH, Wong CS, Hot ST (2007) Gabapentin relieves post-dural puncture headache-a report of two cases. Acta Anaesthesiol Taiwan 45(1):47-51

26. Garza I, Black DF (2006) Persistent primary thunderclap headache responsive to gabapentin. J Headache Pain 7(6):419-421. doi:10.1007/s10194-006-0346-7

27. Coderre TJ, Kuman N, Lefebvre CD, Yu JS (2005) Evidence that gabapentin reduces neuropathic pain by inhibiting the spinal release of glutamate. J Neurochem 94(4):1131-1139

28. Hanna M, O'Brien C, Wilson MC (2008) Prolonged-release oxycodone enhances the effects of existing gabapentin therapy in painful diabetic neuropathy patients. Eur J Pain 12(6):804-813. doi:10.1016/j.ejpain.2007.12.010

29. Matthews EA, Dickenson AH (2002) A combination of gabapentin and morphine mediates enhanced inhibitory effects on dorsal horn neuronal responses in a rat model of neuropathy. Anesthesiology 96:633-640. doi:10.1097/00000542-20020300000020 